\title{
Domínios de atividade física e comportamentos de risco em adolescentes do Nordeste do Brasil: um estudo de monitoramento 2011 e 2016
}

\author{
Domains of physical activity and risk behaviours in adolescents in Northeast Brazil: a monitoring
} study 2011 and 2016

Dominios de la actividad física y comportamientos de riesgo en adolescentes del Noreste de Brasil: un estudio de seguimiento 2011 y 2016

Recebido: 19/01/2021 | Revisado: 23/01/2021 | Aceito: 26/01/2021 | Publicado: 04/02/2021

Ana Claudia Santos Silva Guimarães
ORCID: https://orcid.org/0000-0003-2685-9115
Universidade Federal de Sergipe, Brasil
E-mail: ana20clau@hotmail.com
Lucas Souza Santos
ORCID: https://orcid.org/0000-0003-2172-4596
Universidade Federal de Sergipe, Brasil
E-mail: lucas_souzza@hotmail.com
Davi Soares Santos Ribeiro
ORCID: https://orcid.org/0000-0001-9816-2566
Universidade Federal de Sergipe, Brasil
E-mail: profdavi@live.com
Aldemir Smith Menezes
ORCID: https://orcid.org/0000-0001-5718-6457
Educação, Ciência e Tecnologia de Sergipe, Brasil
E-mail: aldemirsmith@yahoo.com.br

\section{Resumo}

O estudo tem o objetivo de analisar a prevalência e a associação dos domínios de Atividade Física no lazer, na escola e no deslocamento com indicadores de comportamentos de risco em escolares do estado de Sergipe, Brasil, 2011-2016. O estudo trata-se de dois levantamentos epidemiológicos com delineamento transversal realizados em 2011 e 2016 , com amostra representativa de escolares da Rede Estadual de Ensino de Sergipe, composta por 8143 adolescentes $(2011=3992 ; 2016=4151)$, com idade entre 14 e 19 anos. O instrumento utilizado foi o Global School-based Student Health Survey (GSHS). Os dados foram analisados através de estatística descritiva, aplicação do teste do qui-quadrado para análise inferencial e a regressão logística binária bruta e ajustada para associação entre as variáveis. Os resultados apresentaram que o NIAF no Lazer apresentou associação com a exposição ao comportamento sedentário nos dois inquéritos (2011: OR=1,78; IC 95\% 1,56-2,03 / 2016: OR=2,14; IC 95\% 1,88-2,44), com o fato de não fumar tabaco em 2011 (OR=2,11; IC 95\% 1,61-2,79), e com os não usuários de drogas (OR=1,65; IC 95\% 1,19-2,32) e que consumiam baixas porções de frutas no ano de 2016 (OR=1,32; IC 95\% 1,09-1,62). O NIAF Escolar foi associado com o baixo consumo de frutas (2011: OR=2,14; IC 95\% 1,51-3,05/2016: OR=2,61; IC 95\% 1,96-3,50). Conclui-se que foi evidenciado alta prevalência de NIAF entre os inquéritos e os domínios de Atividade Física se associam diferentemente com aspectos comportamentais conforme a exposição dos jovens aos fatores de risco.

Palavras-chave: Domínios de atividade física; Adolescentes; Comportamentos de risco.

\begin{abstract}
The study aims to analyze the prevalence and the association of the domains of Physical Activity in leisure, at school and in commuting with risk behavior indicators in schoolchildren from the state of Sergipe, Brazil, 2011-2016. The study is about two epidemiological surveys with a cross-sectional design carried out in 2011 and 2016 , with a representative sample of students from the State Education Network of Sergipe, composed of 8143 adolescents (2011 $=3992 ; 2016=4151$ ), aged between 14 and 19 years old. The instrument used was the Global School-based Student Health Survey (GSHS). The data were analyzed using descriptive statistics, application of the chi-square test for inferential analysis and crude and adjusted binary logistic regression for association between variables. The results showed that NIAF in Leisure was associated with exposure to sedentary behavior in the two surveys $(2011:$ OR $=1.78$; 95\% CI 1.56-2.03 / 2016: OR = 2.14; 95\% CI 1.88-2.44), with the fact of not smoking tobacco in 2011 (OR = 2.11; $95 \%$ CI 1.61-2.79), and with non-drug users (OR = 1.65; 95\% CI 1.19-2.32) and that consumed low portions of fruit in $2016(\mathrm{OR}=1.32 ; 95 \%$ CI 1.09-1.62). School NIAF was associated with low fruit consumption $(2011:$ OR = 2.14;
\end{abstract}


95\% CI 1.51-3.05 / 2016: OR $=2.61$; 95\% CI 1.96-3.50). It is concluded that there was a high prevalence of NIAF among the surveys and the Physical Activity domains are differently associated with behavioral aspects according to the exposure of young people to risk factors.

Keywords: Physical activity domains; Adolescents; Risk behaviors.

\section{Resumen}

El estudio tiene como objetivo analizar la prevalencia y la asociación de los dominios de la Actividad Física en el ocio, en la escuela y en los desplazamientos con indicadores de conducta de riesgo en escolares del estado de Sergipe, Brasil, 2011-2016. El estudio consta de dos encuestas epidemiológicas con un diseño transversal realizadas en 2011 y 2016 , con una muestra representativa de estudiantes de la Red Estatal de Educación de Sergipe, compuesta por 8143 adolescentes $(2011=3992 ; 2016=4151)$, con edades entre 14 y 19 años. El instrumento utilizado fue la Encuesta Global de Salud de los Estudiantes Escolares (GSHS). Los datos se analizaron mediante estadística descriptiva, aplicación de la prueba de chi-cuadrado para análisis inferencial y regresión logística binaria cruda y ajustada para asociación entre variables. Los resultados mostraron que el NIAF en Ocio se asoció con la exposición al comportamiento sedentario en ambas encuestas (2011: OR = 1,78; IC del 95\%: 1,56-2,03 / 2016: OR = 2,14; IC del 95\% 1,88-2,44), con el hecho de no fumar tabaco en 2011 (OR = 2,11; IC 95\% 1,61-2,79), y con no consumidores de drogas (OR = 1,65 ; IC 95\% 1,19-2,32) y que consumieron porciones bajas de fruta en 2016 (OR = 1,32; IC 95\% 1,09-1,62). El NIAF escolar se asoció con un bajo consumo de fruta (2011: OR = 2,14; IC del 95\%: 1,51-3,05 / 2016: OR = 2,61; IC del 95\%: 1,96$3,50)$. Se concluye que hubo una alta prevalencia de NIAF entre las encuestas y los dominios de Actividad Física se asocian de manera diferente con aspectos conductuales según la exposición de los jóvenes a factores de riesgo.

Palabras clave: Dominios de actividad física; Adolescentes; Conductas de riesgo.

\section{Introdução}

O Nível Insuficiente de Atividade Física (NIAF) é uma preocupação global de saúde pública, pois se caracteriza como uma das principais causas para doenças crônicas não-transmissíveis (Bengoechea, Juan \& Bush, 2013; Jáuregui et al, 2015; WHO 2014), com estimativas de mortalidade crescentes principalmente em países de baixa e média renda (El-ammari \& Bouftini, 2017; Tassitano et al, 2014).

Uma diversidade de fatores, sejam eles socioeconômicos, demográficos, comportamentais, psicológicos, culturais, biológicos e ambientais podem constituir indicadores relevantes associados aos domínios de atividade física no lazer, na escola ou no deslocamento (Bengoechea, Juan \& Bush, 2013; Abildsnes et al, 2017; Peltzer \& Pengpid, 2016).

O período da adolescência é bastante crítico quanto à construção de valores sociais, desenvolvimento físico e adoção de comportamentos relacionados à saúde com tendência a se propagar até a idade adulta (El-ammari \& Bouftini, 2017; Peltzer \& Pengpid, 2016; Lipowski et al, 2016). Nesta fase a prevalência do NIAF a nível mundial é elevada com menos de $20 \%$ dos jovens ativos (Lancet, 2012), conforme as diretrizes internacionais que recomenda um acúmulo de 60 minutos diários de Atividades Físicas Moderadas a Vigorosas (El-ammari \& Bouftini, 2017; Coledam, et. al., 2014) para produzir benefícios à saúde. Portanto, a adoção de um estilo de vida ativo faz-se necessário para a melhoria da aptidão cardiorrespiratória e muscular, biomarcadores metabólicos e saúde mental (Abildsnes et al, 2017; WHO, 2014).

A Atividade Física faz parte de várias ações cotidianas. A duração de sua prática despendidas e acumuladas nos seus diferentes domínios é essencial para se atingir o limiar das recomendações. Por isso é importante promover o transporte ativo para a escola por meio de caminhadas e bicicletas (Jáuregui et al, 2015; Yang et al, 2014), motivar os escolares à participação regular nas aulas de educação física (Abildsnes et al, 2017; Palakshappa et al, 2015) e disseminar intervenções, infraestrutura e incentivo para jogos e exercícios no tempo livre dos jovens (Bengoechea, Juan \& Bush, 2013).

Os adolescentes são movidos por uma necessidade natural de vivenciar novas experiências e emoções que podem ter implicações desfavoráveis na saúde por envolvimento em comportamentos de risco (Lipowski et al, 2016; Hallingberg, Van Goozen \& Moore, 2016), tais como uso do tabaco e álcool, alimentação inadequada e estilo de vida sedentário (Inchley \& Currie, 2016). Além disso, esses fatores de risco tendem a um agregamento preocupante, ou seja, a presença de exposição a um fator aumenta a probabilidade de ocorrência de outros (Tassitano et al, 2014). 
Perante o que foi descrito e considerando a poucas investigações epidemiológicas de representatividade estadual encontradas no Brasil realizadas com adolescentes, estudo desta natureza é relevante para o monitoramento da saúde dos jovens frente à exposição de condutas de risco aliado a tendência elevada de NIAF, a fim de possibilitar planos de ações estratégicas que visem à melhoria da qualidade de vida da população. Tais intervenções podem se difundir de modo simultâneo por programas públicos e nos ambientes da escola e da família.

Neste sentido, o referido estudo objetivou analisar a prevalência e a associação dos domínios de Atividade Física no lazer, na escola e no deslocamento com indicadores de comportamentos de risco em escolares do estado de Sergipe, Brasil, 20112016.

\section{Metodologia}

O estudo caracteriza-se como epidemiológico com delineamento transversal e amostras independentes, realizado com estudantes de 14 a 19 anos de idade matriculados na Rede Estadual de Ensino de Sergipe, Brasil. Possui natureza quantitativa (Pereira A.S. et al., 2018).

Foram analisados dois bancos de dados, de coletas realizadas em 2011 e em 2016, para verificar a prevalência e associação das variáveis da referida pesquisa. O estudo foi intitulado "Tendência Secular sobre Comportamentos de Risco à Saúde em Adolescentes: Estudo CRIS_Adolescentes", desenvolvidos pelo Grupo de Pesquisa em Educação Física e Saúde (GPEFIS) e Instituto Federal de Educação, Ciência e Tecnologia de Sergipe (IFS).

Para o cálculo amostral foi utilizado o software Statcalc do Epiinfo. A estimativa do tamanho da amostra considerou prevalência esperada em 50\%, intervalo de confiança em $95 \%$ e erro tolerável em 5 pontos percentuais. Para análise de associação, além das variáveis acima, também foi considerado um poder estatístico de $80 \%$ e Odds Ratio (OR) de 1,2, sendo necessária uma amostra mínima de 3876 adolescentes para cada inquérito. Acrescentou-se 20\% de escolares à amostra mínima para casos de possíveis perdas.

De acordo com informações do IBGE (Brasil, 2010) o estado de Sergipe é composto por 75 municípios, sendo dividido geograficamente em oito territórios pela Secretaria de Estado do Planejamento. Conforme dados disponíveis na Secretaria de Estado da Educação (Sergipe, 2016), a matrícula do Ensino Médio, realizada em 2010, foi de 58.301 alunos em toda a rede estadual, distribuídos em 155 unidades de ensino. Em 2015, a população matriculada na rede foi de 62.933 alunos, distribuídos em 160 unidades de ensino. Ressalta-se a manutenção do mesmo procedimento metodológico e planejamento amostral para desenvolvimento dos dois inquéritos de pesquisa.

Considerando a amostra mínima necessária para o estudo, sua distribuição realizou-se conforme o porte das unidades de ensino segundo critério do INEP - Instituto Nacional de Estudos e Pesquisas Educacionais Anísio Teixeira (1 = até 199 alunos; $2=200$ a 499 alunos; $3=500+$ alunos). Desse modo, para que todos os territórios fossem contemplados representativamente com os três portes dos colégios, estabeleceu-se como critério o sorteio de $25 \%$ das unidades de ensino do estado, totalizando 39 (2011) e 42 (2016) colégios distribuídos em 30 municípios.

A amostra foi distribuída de forma estratificada e proporcional aos territórios geográficos do estado, com seleção aleatória dos municípios e das unidades de ensino representativas de cada subgrupo. Em seguida, foram sorteadas, por meio de processo aleatório simples, as turmas por série e turno (diurno e noturno), considerando uma média de 20 alunos/turma. Os critérios obedecidos para a inclusão dos estudantes na amostra foram: o aluno estar matriculado no Ensino Médio, estar presente no dia de realização da coleta e responder o questionário adequadamente.

Como instrumento de coleta utilizou-se o GSHS (Global Student Health Survey). Neste modelo constaram questões envolvendo variáveis: sociodemográficas, hábitos alimentares, condições de saúde, higiene, atividades físicas, percepção de 
saúde e estresse, violência, uso de drogas e álcool, comportamentos e relacionamentos. A coleta foi realizada por pesquisadores do GPEFIS devidamente treinados para aplicação do instrumento.

Para proceder com a coleta, foi obtida autorização do Secretário de Estado da Educação, dos diretores das Diretorias Regionais de Educação (DRE’s) e dos diretores das escolas para a participação das instituições na pesquisa. Os estudantes menores de idade receberam um Termo Negativo de Consentimento (Parental Passive Consent Form) para autorização dos pais ou responsáveis, e no dia da coleta de dados eles assinaram um termo de assentimento condicionando voluntariamente sua participação na pesquisa. Os maiores ou iguais a 18 anos assinaram o Termo de Consentimento Livre e Esclarecido.

O primeiro levantamento, em 2011, foi aprovado pelo Comitê de Ética em Pesquisa com Seres Humanos/Hospital Universitário da Universidade Federal de Sergipe (CEP/UFS) sob o protocolo de aprovação n.177/2010; já o projeto de 2016 foi aprovado pelo Comitê de Ética em Pesquisas com Seres Humanos da Plataforma Brasil do Instituto Federal de Sergipe sob o protocolo de aprovação n. 1522876/2016.

Os desfechos deste estudo foram o Nível Insuficiente de Atividade Física (NIAF) no Lazer, na Escola (participação nas aulas de Educação Física) e no deslocamento. Para estimar o NIAF no lazer usou-se a seguinte pergunta: “Qual a atividade de lazer de sua preferência?". Para o NIAF na escola foi questionado: “Durante uma semana típica normal, em quantas aulas de Educação Física você participa?”. O NIAF no deslocamento foi medido por meio das questões: “Durante os últimos 7 dias, em quantos dias você andou a pé ou de bicicleta para ir e voltar da escola?" e "Durante os últimos 7 dias, quanto tempo em média você gastou para ir de casa para a escola e voltar para sua casa (some o tempo que você leva para ir e voltar)?”

Em relação ao NIAF no lazer, os itens assinalados como: praticar esportes, fazer exercícios, nadar ou pedalar foram classificados como lazer ativo; e os itens: jogar dominó ou cartas, assistir TV, jogar videogame, usar o computador e conversar com os amigos foram classificados como lazer inativo. A questão referente ao NIAF na escola, as respostas foram dicotomizadas em nenhuma participação (inativos) e uma ou mais participações (ativos) nas aulas de Educação Física. Em relação ao desfecho de NIAF no deslocamento, os alunos que relataram não ir nenhum dia para a escola a pé ou de bicicleta e aqueles que independente da frequência semanal gastavam menos de 20 minutos de duração, somados o tempo de ida e volta, foram classificados como inativos e o restante como ativos (Santos et al, 2016). As variáveis independentes consideradas foram consumo de álcool, uso de drogas, consumo de tabaco, consumo de frutas, consumo de verduras e comportamento sedentário.

Os dados foram analisados na forma de estatística descritiva expressa em frequências absoluta e relativa. Foi realizada análise inferencial aplicando-se o teste do Qui-quadrado e, para avaliar a associação entre as variáveis, utilizou-se a Regressão Logística Binária Bruta e Ajustada. As variáveis com valor de $\mathrm{p}<0,20$ na análise bruta foram mantidas no modelo ajustado. $\mathrm{O}$ nível de significância adotado foi de $\mathrm{p}<0,05$.

\section{Resultados}

Dados de 9438 (2011=4717; 2016=4721) adolescentes foram coletados; no entanto $1295(2011=725 ; 2016=570)$ foram excluídos por ausência de respostas importantes como o sexo $(2011=03 ; 2016=12)$ e a idade $(2011=02 ; 2016=06)$ e/ou deixarem mais da metade das questões sem resposta $(2011=11 ; 2016=03)$, e aqueles que possuíam idade superior a 19 anos $(2011=709$; 2016=549), totalizando 8143 adolescentes $(2011=3992 ; 2016=4151)$ para a amostra final.

Para os dois inquéritos houve proporção maior de adolescentes do sexo feminino, compreendidos na faixa etária de 1617 anos, estudantes do $1^{\circ}$ ano de Ensino Médio no turno diurno de estudo, residentes na área urbana, de cor da pele parda e com mães de nível baixo de escolaridade. Os índices de reprovação foram elevados, mas com proporção maior nos jovens do sexo masculino em ambos os anos (Tabela 1). A renda familiar foi a única variável que diferenciou em termos percentuais, tornandose mais baixa de 2011 para 2016. 
Tabela 1. Caracterização sociodemográfica da amostra de acordo com os inquéritos 2011 e 2016.

\begin{tabular}{|c|c|c|c|c|}
\hline \multirow[b]{2}{*}{ Variáveis } & \multicolumn{2}{|c|}{ Inquérito 2011} & \multicolumn{2}{|c|}{ Inquérito 2016} \\
\hline & $\begin{array}{c}\text { Masculino } \\
\%(\text { IC } 95 \%)\end{array}$ & $\begin{array}{c}\text { Feminino } \\
\%(\text { IC } 95 \%)\end{array}$ & $\begin{array}{c}\text { Masculino } \\
\%(\text { IC } 95 \%)\end{array}$ & $\begin{array}{c}\text { Feminino } \\
\%(\text { IC } 95 \%)\end{array}$ \\
\hline \multicolumn{5}{|l|}{ Faixa Etária } \\
\hline $14-15$ & $16,8(12,22-21,32)$ & $19,2(15,63-22,76)$ & $15,8(11,51-20,09)$ & $19,9(16,35-23,52)$ \\
\hline $16-17$ & $49,2(45,60-52,71)$ & $53,5(50,81-56,21)$ & $51,6(48,31-54,81)$ & $54,7(52,02-57,42)$ \\
\hline $18-19$ & $34,1(30,01-38,11)$ & $27,3(23,91-30,66)$ & $32,6(28,79-36,46)$ & $25,3(21,87-28,79)$ \\
\hline \multicolumn{5}{|l|}{ Serie do Aluno } \\
\hline $1^{\circ}$ ano & $44,0(40,24-47,71)$ & $39,7(36,58-42,74)$ & $42,6(39,04-46,12)$ & $35,5(32,23-38,67)$ \\
\hline $2^{\circ}$ ano & $32,5(28,41-36,61)$ & $34,4(31,14-37,56)$ & $32,5(28,62-36,30)$ & $33,5(30,26-36,79)$ \\
\hline $3^{\circ}$ ano & $23,5(19,14-27,87)$ & $26,0(22,57-29,38)$ & $25,0(20,90-29,00)$ & $31,0(27,69-34,34)$ \\
\hline \multicolumn{5}{|l|}{ Turno } \\
\hline Diurno & $61,9(58,83-64,99)$ & $69,3(67,12-71,51)$ & $63,8(60,97-66,59)$ & $75,6(73,60-77,56)$ \\
\hline Noturno & $38,1(34,15-42,00)$ & $30,7(27,38-33,97)$ & $36,2(32,48-39,94)$ & $24,4(20,93-27,89)$ \\
\hline \multicolumn{5}{|l|}{ Reprovação } \\
\hline Sim & $56,6(53,26-59,85)$ & $46,0(43,12-48,95)$ & $60,9(57,93-63,80)$ & $49,6(46,74-52,45)$ \\
\hline Não & $43,4(39,68-47,19)$ & $54,0(51,26-56,65)$ & $39,1(35,47-42,78)$ & $50,4(47,56-53,23)$ \\
\hline \multicolumn{5}{|l|}{ Local de Domicílio } \\
\hline Sede (área urbana) & $62,5(59,40-65,60)$ & $60,1(57,58-62,61)$ & $60,4(57,46-63,39)$ & $55,7(53,02-58,41)$ \\
\hline Povoado (área rural) & $37,5(33,48-41,49)$ & $39,9(36,80-42,99)$ & $39,6(35,91-43,23)$ & $44,3(41,26-47,30)$ \\
\hline \multicolumn{5}{|l|}{ Cor da pele } \\
\hline Branca & $20,9(16,48-25,40)$ & $21,6(18,11-25,15)$ & $18,8(14,52-22,97)$ & $19,0(15,34-22,59)$ \\
\hline Preta & $11,0(6,26-15,72)$ & $6,6(2,73-10,42)$ & $9,1(4,68-13,61)$ & $7,0(3,11-10,87)$ \\
\hline Parda & $66,3(63,38-69,20)$ & $70,5(68,39-72,70)$ & $70,0(67,47-72,60)$ & $72,2(70,09-74,33)$ \\
\hline Outro & $1,8(3,20-6,73)$ & $1,2(2,71-5,18)$ & $2,1(2,58-6,69)$ & $1,8(2,17-5,80)$ \\
\hline \multicolumn{5}{|l|}{ Estudo da mãe } \\
\hline E Fund. a & $74,1(71,52-76,75)$ & $77,6(75,65-79,50)$ & $70,2(67,43-72,87)$ & $77,9(75,86-79,85)$ \\
\hline $\mathrm{EM}^{\mathrm{b}}$ Completo & $16,1(11,42-20,85)$ & $15,8(12,06-19,52)$ & $14,7(10,06-19,26)$ & $11,7(7,69-15,65)$ \\
\hline Superior Completo & $9,7(4,83-14,61)$ & $6,6(2,69-10,55)$ & $15,2(10,59-19,76)$ & $10,5(6,45-14,47)$ \\
\hline \multicolumn{5}{|l|}{ Renda Familiar } \\
\hline < 1 Salário Mínimo & $27,4(23,15-31,72)$ & $36,3(33,09-39,49)$ & $51,9(48,53-55,21)$ & $66,2(63,77-68,60)$ \\
\hline 1 - 2 Salários Mínimos & $38,9(34,91-42,78)$ & $40,4(37,29-43,48)$ & $32,5(28,53-36,44)$ & $25,5(21,94-29,10)$ \\
\hline > 2 Salários Mínimos & $33,7(29,60-37,80)$ & $23,3(19,80-26,82)$ & $15,6(11,21-20,06)$ & $8,3(4,31-12,25)$ \\
\hline
\end{tabular}

${ }^{\text {a }}$ Ensino fundamental; ${ }^{\mathrm{b}}$ Ensino Médio

Fonte: Autores (2018).

As prevalências para cada desfecho são diferentes em relação às variáveis de comportamentos de risco (tabela 2). O NIAF no lazer obteve prevalência geral de 47,8\% e 43, 1\%, nas aulas de Educação Física de 25,4\% e 29,6\% e no deslocamento de $70,7 \%$ e $75 \%$, em 2011 e 2016 respectivamente.

O NIAF no lazer, em 2011, foi mais prevalente entre os escolares que consumiam álcool, mas não usavam tabaco ou drogas; que comiam frutas de forma esporádica, verduras frequentemente 1 a 2 vezes por dia e mantinham 3 horas ou mais de comportamento sedentário diários. Em 2016, essa prevalência se deu entre os não adeptos do uso de álcool, tabaco e drogas, com baixo consumo de frutas e verduras e com elevado comportamento sedentário.

O NIAF na escola apontou predomínio maior para os que tinham usado drogas, não fumavam tabaco, não haviam comido frutas e verduras no último mês e tinham baixa exposição ao comportamento sedentário. Já em 2016, a predominância foi maior nos que consumiam álcool, drogas e tabaco, não ingeriam frutas e verduras e eram mais expostos ao comportamento sedentário.

A prevalência do NIAF de deslocamento em 2011 ocorreu entre os consumidores de álcool e tabaco, não usuários de drogas, com baixa ingestão de frutas e verduras e poucas horas de exposição ao comportamento sedentário. Em 2016, a situação se modificou apenas quanto ao consumo de álcool, tabaco, frutas e verduras. 
Observou-se que para todos os comportamentos o NIAF no lazer foi o único desfecho mais predominante em 2011 do que em 2016; ao contrário dos NIAFs na escola e no deslocamento, pois estes obtiveram maiores prevalências no ano de 2016 para cada fator de risco.

A tabela 3 apresenta a análise de Regressão Logística Bruta do NIAF nos diferentes contextos. Em 2011, as variáveis que se associaram ao desfecho do Lazer foram o uso de drogas, de tabaco e o comportamento sedentário. Já a que se associou ao NIAF escolar foi o consumo de frutas. Quanto ao NIAF no deslocamento houve associação somente com o consumo de tabaco.

Em 2016 houve associação com o NIAF no lazer em relação ao uso de drogas, consumo de frutas, verduras e comportamento sedentário. O NIAF na escola se associou com o consumo de frutas e verduras. Com o NIAF no deslocamento a associação se deu com o uso do tabaco e comportamento sedentário.

Na análise de Regressão Ajustada (tabela 4), observou-se que no ano de 2011, os escolares que não fumavam tabaco e aqueles que eram mais expostos ao comportamento sedentário, possuíam mais chances de serem inativos no lazer. Já no ano de 2016, os grupos que não usavam drogas, tinham baixa ingestão de frutas e 3 horas ou mais de exposição ao comportamento sedentário, possuíam chances maiores de NIAF.

Adolescentes que não tinham alimentação rica em frutas possuíam mais chances de serem inativos na escola, após ajuste das variáveis para o ano de 2011, e as mesmas chances se repetem em relação aos resultados de 2016. Quanto ao NIAF de deslocamento, após as variáveis serem ajustadas, os escolares que não usavam tabaco são os que possuíam mais chances de inatividade nos dois anos de coleta. 
Tabela 2. Prevalência do Nível Insuficiente de Atividade Física no Lazer, nas Aulas de Educação Física e no Deslocamento Casa-Escola, 2011 e 2016.

\begin{tabular}{|c|c|c|c|c|c|c|c|c|c|c|c|c|}
\hline \multirow{3}{*}{ Variáveis } & \multicolumn{6}{|c|}{ NIAF a 2011} & \multicolumn{6}{|c|}{ NIAF 2016} \\
\hline & \multicolumn{2}{|r|}{ Lazer } & \multicolumn{2}{|r|}{ Escola } & \multicolumn{2}{|c|}{ Deslocamento } & \multicolumn{2}{|r|}{ Lazer } & \multicolumn{2}{|r|}{ Escola } & \multicolumn{2}{|c|}{ Deslocamento } \\
\hline & $\%$ & IC $(95 \%)$ & $\%$ & IC $(95 \%)$ & $\%$ & IC $(95 \%)$ & $\%$ & IC $(95 \%)$ & $\%$ & IC $(95 \%)$ & $\%$ & IC $(95 \%)$ \\
\hline \multicolumn{13}{|l|}{ Consumo de Álcool } \\
\hline Sim & 48,4 & $45,60-51,27$ & 24,4 & $20,92-27,81$ & 71,3 & $69,10-73,43$ & 42,9 & $38,80-47,02$ & 31,3 & $26,76-35,79$ & 73,9 & $71,06-76,76$ \\
\hline Não & 46,8 & $43,10-50,51$ & 24,4 & $19,98-28,84$ & 69,8 & $66,91-72,63$ & 43,2 & $40,45-46,04$ & 29,1 & $25,98-32,22$ & 75,4 & $73,56-77,31$ \\
\hline \multicolumn{13}{|l|}{ Uso de Drogas } \\
\hline Sim & 39,4 & $29,10-49,63$ & 25,0 & $13,56-36,44$ & 67,3 & $59,54-75,08$ & 34,1 & $21,92-46,32$ & 34,9 & $22,75-47,07$ & 72,5 & $64,37-80,63$ \\
\hline Não & 48,2 & $45,87-50,50$ & 24,3 & $21,54-27,15$ & 70,9 & $69,09-72,63$ & 43,6 & $41,20-45,98$ & 29,4 & $26,68-32,03$ & 75,2 & $73,54-76,78$ \\
\hline \multicolumn{13}{|l|}{ Consumo de Tabaco } \\
\hline Não & 49,2 & $46,81-51,62$ & 24,6 & $21,68-27,55$ & 72,2 & $70,35-73,98$ & 43,5 & $40,98-45,96$ & 29,6 & $26,81-32,38$ & 75,5 & $73,82-77,17$ \\
\hline Sim & 30,2 & $20,40-40,04$ & 21,9 & $11,31-32,40$ & 55,9 & $47,72-63,99$ & 41,5 & $30,90-52,10$ & 32,3 & $20,86-43,78$ & 67,0 & $58,87-75,16$ \\
\hline \multicolumn{13}{|l|}{ Consumo de Frutas } \\
\hline Não comeu & 46,1 & $35,28-56,86$ & 36,2 & $24,34-48,07$ & 65,7 & $56,94-74,45$ & 45,5 & $37,12-53,85$ & 45,1 & $36,63-53,54$ & 72,8 & $66,64-78,88$ \\
\hline$>1 \mathrm{x} / \mathrm{dia}$ & 49,6 & $44,40-54,76$ & 28,8 & $22,66-35,00$ & 71,8 & $67,83-75,77$ & 46,0 & $41,18-50,88$ & 34,0 & $28,59-39,37$ & 74,1 & $70,61-77,49$ \\
\hline 1 a $2 x / d i a$ & 48,8 & $45,60-52,00$ & 23,8 & $19,85-27,69$ & 70,2 & $67,73-72,73$ & 44,4 & $40,98-47,74$ & 28,5 & $24,05-32,31$ & 76,1 & $73,85-78,36$ \\
\hline$\geq 3 \mathrm{x} / \mathrm{dia}$ & 45,4 & $40,96-49,75$ & 20,6 & $15,33-25,96$ & 71,7 & $68,52-74,97$ & 38,3 & $33,53-43,08$ & 23,8 & $18,46-29,08$ & 74,4 & $71,29-77,60$ \\
\hline \multicolumn{13}{|l|}{ Consumo de Verduras } \\
\hline Não comeu & 45,7 & $38,46-52,85$ & 27,8 & $19,48-36,16$ & 72,5 & $67,23-77,71$ & 47,5 & $39,85-55,19$ & 34,4 & $25,83-42,97$ & 72,4 & $66,73-78,02$ \\
\hline$>1 \mathrm{x} / \mathrm{dia}$ & 47,5 & $42,02-52,95$ & 26,0 & $19,55-32,52$ & 68,7 & $64,31-73,02$ & 42,9 & $37,50-48,36$ & 31,6 & $25,68-37,57$ & 73,4 & $69,58-77,17$ \\
\hline 1 a $2 x / d i a$ & 49,1 & $46,12-52,04$ & 23,2 & $19,57-26,87$ & 71,7 & $69,43-73,93$ & 43,9 & $40,87-46,98$ & 29,6 & $26,23-33,07$ & 76,6 & $74,61-78,64$ \\
\hline$\geq 3 \mathrm{x} / \mathrm{dia}$ & 45,0 & $39,15-50,95$ & 24,0 & $16,99-30,93$ & 67,8 & $63,14-72,38$ & 38,9 & $33,10-44,76$ & 24,9 & $18,38-31,33$ & 73,6 & $69,65-77,52$ \\
\hline \multicolumn{13}{|l|}{$\begin{array}{l}\text { Comportamento } \\
\text { Sedentário }\end{array}$} \\
\hline$\leq 2 \mathrm{~h} / \mathrm{dia}$ & 41,1 & $37,86-44,40$ & 24,5 & $20,82-28,25$ & 71,2 & $68,85-73,54$ & 34,9 & $31,60-38,25$ & 29,1 & $25,60-32,55$ & 76,4 & $74,37-78,46$ \\
\hline$\geq 3 \mathrm{~h} / \mathrm{dia}$ & 55,8 & $52,70-58,82$ & 24,2 & $20,16-28,20$ & 71,1 & $67,54-72,65$ & 53,3 & $50,20-56,45$ & 30,3 & $26,51-34,15$ & 73,6 & $71,18-75,98$ \\
\hline
\end{tabular}

a Nível Insuficiente de Atividade Física

Fonte: Autores (2018). 
Tabela 3. Análise de regressão logística bruta do Nível Insuficiente de Atividade Física em seus domínios associados a fatores comportamentais, 2011 e 2016.

\begin{tabular}{|c|c|c|c|c|c|c|c|}
\hline \multirow[b]{2}{*}{ Variáveis } & \multirow[b]{2}{*}{ Categorias } & \multicolumn{3}{|c|}{ NIAF $^{\text {a }}-2011$} & \multicolumn{3}{|c|}{ NIAF - 2016} \\
\hline & & $\begin{array}{c}\text { OR (IC } \\
95 \%) \\
\text { Lazer }\end{array}$ & $\begin{array}{c}\text { OR (IC } \\
95 \%) \\
\text { Escola }\end{array}$ & $\begin{array}{l}\text { OR (IC 95\%) } \\
\text { Deslocamento }\end{array}$ & $\begin{array}{c}\text { OR (IC } \\
95 \%) \\
\text { Lazer }\end{array}$ & $\begin{array}{c}\text { OR (IC } \\
95 \%) \\
\text { Escola }\end{array}$ & $\begin{array}{l}\text { OR (IC 95\%) } \\
\text { Deslocamento }\end{array}$ \\
\hline \multirow[t]{2}{*}{$\begin{array}{l}\text { Consumo de } \\
\text { Álcool }\end{array}$} & Sim & $\begin{array}{c}1,07(0,94- \\
1,21)\end{array}$ & $\begin{array}{c}0,99(0,86- \\
1,16)\end{array}$ & $\begin{array}{c}1,07(0,93- \\
1,24)\end{array}$ & $\begin{array}{c}0,99(0,86- \\
1,13)\end{array}$ & $\begin{array}{c}1,10(0,96- \\
1,28)\end{array}$ & $\begin{array}{c}0,92(0,79- \\
1,08)\end{array}$ \\
\hline & Não & 1 & 1 & 1 & 1 & 1 & 1 \\
\hline P valor & & 0,322 & 0,976 & 0,327 & 0,841 & 0,157 & 0,305 \\
\hline \multirow[t]{2}{*}{ Uso de Drogas } & Sim & 1 & $\begin{array}{c}1,03(0,76- \\
1,42)\end{array}$ & $\begin{array}{c}0,84(0,61- \\
1,14)\end{array}$ & 1 & $\begin{array}{c}1,29(0,93- \\
1,78)\end{array}$ & $\begin{array}{c}0,87(0,61- \\
1,24)\end{array}$ \\
\hline & Não & $\begin{array}{c}1,43(1,08- \\
1,89)\end{array}$ & 1 & 1 & $\begin{array}{c}1,49(1,08- \\
2,06)\end{array}$ & 1 & 1 \\
\hline P valor & & 0,011 & 0,825 & 0,275 & 0,015 & 0,122 & 0,447 \\
\hline $\begin{array}{l}\text { Consumo de } \\
\text { Tabaco }\end{array}$ & Não & $\begin{array}{c}2,23(1,72- \\
2,92)\end{array}$ & $\begin{array}{c}1,16(0,87- \\
1,57)\end{array}$ & $\begin{array}{c}2,04(1,58- \\
2,65)\end{array}$ & $\begin{array}{c}1,08(0,81- \\
1,45)\end{array}$ & $\begin{array}{c}0,88(0,65- \\
1,20)\end{array}$ & $\begin{array}{c}1,51(1,11- \\
2,07)\end{array}$ \\
\hline & Sim & 1 & 1 & 1 & 1 & 1 & 1 \\
\hline P valor & & $>0,001$ & 0,310 & $>0,001$ & 0,585 & 0,415 & 0,009 \\
\hline \multirow[t]{4}{*}{$\begin{array}{l}\text { Consumo de } \\
\text { Frutas }\end{array}$} & Não comeu & $\begin{array}{c}1,02(0,75- \\
1,41)\end{array}$ & $\begin{array}{c}2,18(1,55- \\
3,07)\end{array}$ & $\begin{array}{c}0,75(0,54- \\
1,06)\end{array}$ & $\begin{array}{c}1,34(1,04- \\
1,74)\end{array}$ & $\begin{array}{c}2,63(2,01- \\
3,45)\end{array}$ & $\begin{array}{c}0,91(0,68- \\
1,24)\end{array}$ \\
\hline & $>1 \mathrm{x} / \mathrm{dia}$ & $\begin{array}{c}1,18(0,98- \\
1,43)\end{array}$ & $\begin{array}{c}1,55(1,25- \\
1,94)\end{array}$ & $\begin{array}{c}1,00(0,81- \\
1,24)\end{array}$ & $\begin{array}{c}1,37(1,14- \\
1,65)\end{array}$ & $\begin{array}{c}1,65(1,35- \\
2,02)\end{array}$ & $\begin{array}{c}0,98(0,79- \\
1,21)\end{array}$ \\
\hline & 1 a $2 x / d i a$ & $\begin{array}{c}1,14(0,99- \\
1,33)\end{array}$ & $\begin{array}{c}1,19(1,00- \\
1,44)\end{array}$ & $\begin{array}{c}0,92(0,79- \\
1,10)\end{array}$ & $\begin{array}{c}1,28(1,10- \\
1,50)\end{array}$ & $\begin{array}{c}1,27(1,07- \\
1,52)\end{array}$ & $\begin{array}{c}1,09(0,91- \\
1,31)\end{array}$ \\
\hline & $\geq 3 x /$ dia & 1 & 1 & 1 & 1 & 1 & 1 \\
\hline P valor & & 0,212 & $>0,001$ & 0,359 & 0,002 & $>0,001$ & 0,477 \\
\hline \multirow[t]{4}{*}{$\begin{array}{l}\text { Consumo de } \\
\text { Verduras }\end{array}$} & Não comeu & $\begin{array}{c}1,02(0,80- \\
1,32)\end{array}$ & $\begin{array}{c}1,22(0,92- \\
1,63)\end{array}$ & $\begin{array}{c}1,25(0,94- \\
1,66)\end{array}$ & $\begin{array}{c}1,42(1,09- \\
1,84)\end{array}$ & $\begin{array}{c}1,58(1,20- \\
2,10)\end{array}$ & $\begin{array}{c}0,94(0,70- \\
1,26)\end{array}$ \\
\hline & $>1 \mathrm{x} / \mathrm{dia}$ & $\begin{array}{c}1,10(0,88- \\
1,37)\end{array}$ & $\begin{array}{c}1,11(0,87- \\
1,44)\end{array}$ & $\begin{array}{c}1,04(0,82- \\
1,33)\end{array}$ & $\begin{array}{c}1,18(0,96- \\
1,46)\end{array}$ & $\begin{array}{c}1,39(1,11- \\
1,76)\end{array}$ & $\begin{array}{c}0,98(0,78- \\
1,26)\end{array}$ \\
\hline & 1 a $2 x / d i a$ & $\begin{array}{c}1,17(0,98- \\
1,41)\end{array}$ & $\begin{array}{c}0,96(0,78- \\
1,19)\end{array}$ & $\begin{array}{c}1,20(0,99- \\
1,47)\end{array}$ & $\begin{array}{c}1,22(1,03- \\
1,46)\end{array}$ & $\begin{array}{c}1,27(1,05- \\
1,55)\end{array}$ & $\begin{array}{c}1,17(0,96- \\
1,44)\end{array}$ \\
\hline & $\geq 3 \mathrm{x} / \mathrm{dia}$ & 1 & 1 & 1 & 1 & 1 & 1 \\
\hline P valor & & 0,253 & 0,156 & 0,154 & 0,041 & 0,005 & 0,112 \\
\hline \multirow{2}{*}{$\begin{array}{l}\text { Comportamento } \\
\text { Sedentário }\end{array}$} & $\leq 2 \mathrm{~h} / \mathrm{dia}$ & 1 & $\begin{array}{c}1,01(0,88- \\
1,18)\end{array}$ & $\begin{array}{c}1,05(0,92- \\
1,21)\end{array}$ & 1 & $\begin{array}{c}0,94(0,82- \\
1,08)\end{array}$ & $\begin{array}{c}1,16(1,01- \\
1,34)\end{array}$ \\
\hline & $\geq 3 \mathrm{~h} / \mathrm{dia}$ & $\begin{array}{c}1,80(1,59- \\
2,05)\end{array}$ & 1 & 1 & $\begin{array}{c}2,12(1,88- \\
2,41)\end{array}$ & 1 & 1 \\
\hline P valor & & $>0,001$ & 0,798 & 0,460 & $>0,001$ & 0,382 & 0,041 \\
\hline
\end{tabular}

${ }^{a}$ Nível Insuficiente de Atividade Física

Fonte: Autores (2018). 
Tabela 4. Análise de regressão logística ajustada do Nível Insuficiente de Atividade Física em seus domínios associados a fatores comportamentais, 2011 e 2016.

\begin{tabular}{|c|c|c|c|c|c|c|c|}
\hline \multirow[b]{2}{*}{ Variáveis } & \multirow[b]{2}{*}{ Categorias } & \multicolumn{3}{|c|}{ NIAF $^{\text {a }}$ - 2011} & \multicolumn{3}{|c|}{ NIAF - 2016} \\
\hline & & $\begin{array}{c}\text { OR (IC } \\
95 \%) \\
\text { Lazer }\end{array}$ & $\begin{array}{c}\text { OR (IC } \\
95 \%) \\
\text { Escola }\end{array}$ & $\begin{array}{l}\text { OR (IC 95\%) } \\
\text { Deslocamento }\end{array}$ & $\begin{array}{c}\text { OR (IC } \\
95 \%) \\
\text { Lazer }\end{array}$ & $\begin{array}{c}\text { OR (IC } \\
95 \%) \\
\text { Escola }\end{array}$ & $\begin{array}{l}\text { OR (IC 95\%) } \\
\text { Deslocamento }\end{array}$ \\
\hline \multirow[t]{2}{*}{$\begin{array}{l}\text { Consumo de } \\
\text { Álcool }\end{array}$} & Sim & -- & -- & -- & -- & $\begin{array}{c}1,12(0,97- \\
1,31)\end{array}$ & -- \\
\hline & Não & -- & -- & -- & -- & 1 & -- \\
\hline P valor & & -- & -- & -- & -- & 0,128 & -- \\
\hline \multirow[t]{2}{*}{ Uso de Drogas } & Sim & $\begin{array}{c}0,83(0,62- \\
1,13)\end{array}$ & -- & -- & 1 & $\begin{array}{c}1,25(0,89- \\
1,76)\end{array}$ & -- \\
\hline & Não & 1 & -- & -- & $\begin{array}{c}1,65(1,19- \\
2,32)\end{array}$ & 1 & -- \\
\hline P valor & & 0,242 & -- & -- & 0,003 & 0,187 & -- \\
\hline $\begin{array}{l}\text { Consumo de } \\
\text { Tabaco }\end{array}$ & Não & $\begin{array}{c}2,11(1,61- \\
2,79)\end{array}$ & -- & $\begin{array}{l}2,05(1,58- \\
2,67)\end{array}$ & -- & -- & $\begin{array}{c}1,38(1,00- \\
1,91)\end{array}$ \\
\hline & Sim & 1 & -- & 1 & -- & -- & 1 \\
\hline P valor & & $>0,001$ & -- & $>0,001$ & -- & -- & 0,050 \\
\hline \multirow[t]{4}{*}{$\begin{array}{l}\text { Consumo de } \\
\text { Frutas }\end{array}$} & Não comeu & -- & $\begin{array}{c}2,14(1,51- \\
3,05)\end{array}$ & -- & $\begin{array}{c}1,26(0,95- \\
1,69)\end{array}$ & $\begin{array}{c}2,61(1,96- \\
3,50)\end{array}$ & -- \\
\hline & $>1 \mathrm{x} / \mathrm{dia}$ & -- & $\begin{array}{c}1,57(1,25- \\
1,98)\end{array}$ & -- & $\begin{array}{c}1,32(1,09- \\
1,62)\end{array}$ & $\begin{array}{c}1,56(1,26- \\
1,95)\end{array}$ & -- \\
\hline & 1 a $2 x / d i a$ & -- & $\begin{array}{c}1,21(1,01- \\
1,47)\end{array}$ & -- & $\begin{array}{c}1,23(1,04- \\
1,46)\end{array}$ & $\begin{array}{c}1,22(1,01- \\
1,47)\end{array}$ & -- \\
\hline & $\geq 3 \mathrm{x} / \mathrm{dia}$ & -- & 1 & -- & 1 & 1 & -- \\
\hline P valor & & -- & $>0,001$ & -- & $\mathbf{0 , 0 3 1}$ & $>0,001$ & -- \\
\hline \multirow[t]{4}{*}{$\begin{array}{l}\text { Consumo de } \\
\text { Verduras }\end{array}$} & Não comeu & -- & $\begin{array}{c}1,04(0,78- \\
1,41)\end{array}$ & $\begin{array}{c}1,34(0,99- \\
1,80)\end{array}$ & $\begin{array}{c}1,27(0,95- \\
1,69)\end{array}$ & $\begin{array}{c}1,21(0,89- \\
1,65)\end{array}$ & $\begin{array}{c}0,97(0,71- \\
1,34)\end{array}$ \\
\hline & $>1 \mathrm{x} / \mathrm{dia}$ & -- & $\begin{array}{c}0,94(0,72- \\
1,23)\end{array}$ & $\begin{array}{c}1,06(0,83- \\
1,37)\end{array}$ & $\begin{array}{c}1,07(0,85- \\
1,35)\end{array}$ & $\begin{array}{c}1,13(0,88- \\
1,46)\end{array}$ & $\begin{array}{c}0,99(0,77- \\
1,29)\end{array}$ \\
\hline & 1 a $2 x / d i a$ & -- & $\begin{array}{c}0,88(0,71- \\
1,10)\end{array}$ & $\begin{array}{c}1,18(0,96- \\
1,46)\end{array}$ & $\begin{array}{c}1,16(0,96- \\
1,40)\end{array}$ & $\begin{array}{c}1,17(0,96- \\
1,45)\end{array}$ & $\begin{array}{c}1,23(1,00- \\
1,52)\end{array}$ \\
\hline & $\geq 3 \mathrm{x} / \mathrm{dia}$ & -- & 1 & 1 & 1 & 1 & 1 \\
\hline P valor & & -- & 0,447 & 0,172 & 0,262 & 0,452 & 0,057 \\
\hline \multirow{2}{*}{$\begin{array}{l}\text { Comportamento } \\
\text { Sedentário }\end{array}$} & $\leq 2 \mathrm{~h} / \mathrm{dia}$ & 1 & -- & -- & 1 & -- & $\begin{array}{c}1,12(0,96- \\
1,31)\end{array}$ \\
\hline & $\geq 3 \mathrm{~h} / \mathrm{dia}$ & $\begin{array}{c}1,78(1,56- \\
2,03)\end{array}$ & -- & -- & $\begin{array}{c}2,14(1,88- \\
2,44)\end{array}$ & -- & 1 \\
\hline P valor & & $>0,001$ & -- & -- & $>0,001$ & -- & 0,145 \\
\hline
\end{tabular}

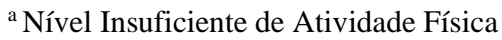

Fonte: Autores (2018).

\section{Discussão}

O referido estudo demonstrou elevada prevalência de NIAF em diferentes domínios relacionados a comportamentos de risco nos adolescentes. Verificou-se associação do NIAF no lazer com os não fumantes em 2011; com os não usuários de drogas, mas com baixo consumo de frutas em 2016; e com os expostos ao comportamento sedentário nos dois inquéritos.

O NIAF na escola se associou com o baixo consumo de frutas e o NIAF no deslocamento com os não fumantes tanto em 2011 como em 2016. O consumo de álcool foi o único comportamento não significativo para os desfechos, assim como em outros estudos (Bengoechea, Juan \& Bush, 2013; deRuiter et al, 2016), pois quantidades moderadas são socialmente aceitáveis pela população e não demonstra influência sobre o nível de atividade física. 
No domínio do lazer, em 2011 foi constatado que os não fumantes eram mais propensos ao NIAF, o que contraria grande parte das pesquisas realizadas no Sudoeste da Espanha (Bengoechea, Juan \& Bush, 2013), Canadá (deRuiter et al, 2016), Noruega (Wichstrøm, von Soest \& Kvalem, 2013) e Alemanha (Höpker, Lampert \& Spallek, 2014), as quais indicam que o hábito de fumar é preditor de baixos NIAF no lazer. Tais estudos justificam que o fumo está relacionado a uma capacidade aeróbica reduzida (Wichstrøm, von Soest \& Kvalem, 2013) e a Atividade física pode impor influência favorável a este comportamento de risco já que tem sido demonstrada a sua capacidade de diminuir o desejo do fumo e minimizar os sintomas de sua retirada ao organismo (deRuiter et al, 2016).

No entanto, estudo brasileiro mostra resultados condizentes com os desta pesquisa, mostrando relação inversa entre exposição ao tabagismo e NIAF no lazer (Bezerra et al, 2015). Outro estudo evidenciou que adolescentes com bons níveis de Atividade Física apresentaram consumo elevado de várias substâncias incluindo o uso do tabaco (de Oliveira, Andrade \& De Micheli, 2016). Uma das possíveis explicações seja o vínculo entre tabagismo e etilismo, como um fator levando à adesão ao uso do outro em eventos de lazer sociais, onde as atividades físicas e recreativas também estejam presentes, especialmente nos fins de semana (Bezerra et al, 2015).

Além disso, a prática regular de atividades físicas pode não ser fator de proteção ao envolvimento de comportamentos de risco (Lipowski et al, 2016; de Oliveira, Andrade \& De Micheli, 2016), especialmente se realizadas longe de casa em período de lazer, sem presença familiar, suscetível ao influxo de outros contextos comunitários e psicossociais (Lipowski et al, 2016).

O uso do tabaco é a principal causa de morte evitável e um grande obstáculo ao desenvolvimento dos países (WHO, 2014). Ele pode ser reflexo da junção de comportamentos insalubres (Bengoechea, Juan \& Bush, 2013) e influência de colegas, não propícios ou adeptos à disseminação de Atividades Físicas e/ou Esportivas bem como de sua importância para a saúde (Wichstrøm, von Soest \& Kvalem, 2013; Höpker, Lampert \& Spallek, 2014). Verifica-se uma tendência negativa desse comportamento de risco que pode ser consequência da difusão de políticas públicas e sociais destinadas ao controle e erradicação dessa conduta visualizada no país nas últimas décadas, o que também é demonstrado em outros países (deRuiter et al, 2016; Sousa, José \& Barbosa, 2013).

No ano de 2016 não se verificou associação com o consumo de tabaco, mas sim entre os estudantes que não usavam drogas com maior probabilidade ao NIAF no lazer. Outro estudo brasileiro identificou resultados semelhantes com um maior nível de atividade física relacionado ao uso de drogas nos últimos 30 dias, sendo uma das razões possíveis, a agregação de comportamentos nocivos à saúde (de Rezende et al, 2014).

O baixo consumo de frutas também foi associado ao NIAF no lazer em 2016, o que condiz com trabalhos oriundos da Suécia (Winkvist, et. al, 2015), Espanha (Alonso-Fernández, et al, 2015), Noruega (Wichstrøm, von Soest \& Kvalem, 2013), Alemanha (Höpker, Lampert \& Spallek, 2014) e Brasil (Sousa, José \& Barbosa, 2013; de Rezende et al, 2014). Os adolescentes com um alto escorem nutricional baseado em alimentos saudáveis, que inclui frutas, hortaliças e vegetais, em geral são mais ativos durante seu tempo de Lazer (Wichstrøm, von Soest \& Kvalem, 2013; de Rezende et al, 2014), além de apresentarem baixas taxas de sobrepeso e obesidade (Winkvist, et. al, 2015) e menor incidência de morbidades de risco cardiovascular (Sousa, José \& Barbosa, 2013; Alonso-Fernández et al, 2015).

Quanto ao comportamento sedentário verificou-se que os jovens que possuíam 3 horas ou mais de exposição diária foram os mais prováveis ao NIAF no lazer nos dois inquéritos. Tal resultado é condizente com investigações realizadas no Brasil (de Rezende et al, 2014), Nepal (Paudel et al, 2014), Espanha (Alonso-Fernández et al, 2015), Alemanha (Höpker, Lampert \& Spallek, 2014) e Estados Unidos (Bassett et al, 2015). Contudo, pesquisas advindas da Suécia (Winkvist et al, 2015) e Escócia (McNeill et al, 2017) apresentaram resultados diferentes deste estudo, pois o primeiro mostrou elevada prevalência dos jovens em atividade físicas de lazer com baixo uso do tempo de tela, enquanto o segundo evidenciou tendência negativa das atividades físicas de lazer entre dois estudos transversais sem mudanças no uso do tempo de tela. 
Os entretenimentos eletrônicos tais como televisão, videogames, computadores e aparelhos celulares são altamente acessíveis e atraentes para o período de lazer entre os jovens desencadeando mais tempo sentado e tornando-os mais suscetíveis ao desinteresse pelas atividades físicas, mesmo quando alternativas destas práticas se encontram disponíveis (Alonso-Fernández et al, 2015; Paudel et al, 2014; Biddle, Petrolini \& Pearson, 2014).

Em contraste também, a falta de estratégias de promoção de atividades físicas ao ar livre, de intervenções e acesso fácil a espaços públicos, pouca infraestrutura, insegurança devido à violência urbana (de Rezende et al, 2014) e relações interpessoais da família e da comunidade que não motivam a um comportamento ativo (Bassett et al, 2015) são fatores que incitam a um estilo de vida sedentário.

O NIAF na escola foi associado com o baixo consumo de frutas nos dois anos de coleta. Resultados semelhantes foram encontrados em trabalhos da Noruega (Abildsnes et al, 2017) e no Brasil (Tassitano et al, 2014). Os jovens que não participam ou são menos disciplinados nas aulas de Educação Física tendem a uma agregação de comportamentos de risco, apresentam alimentação irregular (Abildsnes et al, 2017) e consumo inadequado de frutas, legumes e vegetais (Tassitano et al, 2014). Isso é justificável, pois evidências indicam que a Educação Física Escolar é um meio importante de conhecimento sobre hábitos saudáveis podendo intervir diretamente no desenvolvimento de uma melhor qualidade de vida durante e pós-adolescência (Tassitano et al, 2014; Bassett et al, 2015).

O NIAF no deslocamento foi associado com os não fumantes. Há uma dificuldade de pesquisas que indique uma relação direta deste comportamento de risco com o fato de o jovem se deslocar ou não de modo ativo para a escola. No entanto, trabalho realizado na Finlândia (Yang et al, 2014) mostrou que os participantes com deslocamento ativo eram menos propensos a fumar do que seus homólogos passivos. Possivelmente, esses resultados sejam devido a características peculiares da população investigada quanto ao estilo de vida, demografia ou aspectos socioeconômicos.

\section{Conclusão}

Os escolares mantiveram prevalências elevadas de NIAF, principalmente nos domínios do lazer e deslocamento, entre os inquéritos. Verificou-se que determinados comportamentos de risco se associam às predominâncias dos NIAFs. O NIAF no lazer apresentou associação com a exposição ao comportamento sedentário nos dois inquéritos, com o fato de não fumar tabaco em 2011, e com os não usuários de drogas e que consumiam baixas porções de frutas no ano de 2016. O NIAF escolar foi associado com o baixo consumo de frutas e o NIAF no deslocamento com os não fumantes.

Os resultados deste estudo podem ter implicações relevantes para auxiliar políticas públicas de saúde em ações de controle e redução dos comportamentos de risco, suscitando a adesão de hábitos saudáveis, bem como a promoção de estratégias de incentivo à atividade física entre os jovens, através de programas sociais, do ambiente familiar e da escola.

\section{Referências}

Abildsnes, E., Rohde, G., Berntsen, S., \& Stea, T. H. (2017). Fun, influence and competence — a mixed methods study of prerequisites for high school students' participation in physical education. BMC Public Health, 17(1), 1-12.

Alonso-Fernández, N., Jiménez-García, R., Alonso-Fernández, L., Hernández-Barrera, V., \& Palacios-Ceña, D. (2015). Health factors related to physical activity among children and adolescents: Results from Spanish National Health Surveys 2006 and 2011/12. Journal for Specialists in Pediatric Nursing, 20(3), 193-202.

Bassett, D. R., John, D., Conger, S. A., Fitzhugh, E. C., \& Coe, D. P. (2015). Trends in physical activity and sedentary behaviors of United States youth. Journal of physical activity and health, 12(8), 1102-1111.

Bengoechea, E. G., Juan, F. R., \& Bush, P. L. (2013). Delving into the social ecology of leisure-time physical activity among adolescents from south eastern Spain. Journal of Physical Activity and Health, 10(8), 1136-1144.

Bezerra, J., Lopes, A. S., Hardmam, C. M., Tassitano, R. M., Tenório, M. C. M., \& de Barros, M. V. G. (2015). Consumo de bebidas alcoólicas e tabagismo: associação com inatividade física no lazer e comportamento sedentário. Revista Andaluza de Medicina del Deporte, 8(1), 1-6. 
Biddle, S. J., Petrolini, I., \& Pearson, N. (2014). Interventions designed to reduce sedentary behaviours in young people: a review of reviews. British journal of sports medicine, 48(3), 182-186.

Brasil, I. B. G. E. (2010). Instituto Brasileiro de geografia e Estatística. Censo demográfico, 2010.

Coledam, D. H. C., Ferraiol, P. F., Júnior, R. P., Ribeiro, E. A. G., Ferreira, M. A. C., \& de Oliveira, A. R. (2014). Agreement between two cutoff points for physical activity and associated factors in young individuals. Revista Paulista de Pediatria (English Edition), 32(3), 215-222.

de Oliveira Pinheiro, B., Andrade, A. L. M., \& De Micheli, D. (2016). Relação entre os níveis de atividade física e qualidade de vida no uso de drogas em adolescentes. SMAD, Revista Electrónica en Salud Mental, Alcohol y Drogas, 12(3), 171-180.

de Rezende, L. F. M., Azeredo, C. M., Canella, D. S., Claro, R. M., de Castro, I. R. R., Levy, R. B., \& do Carmo Luiz, O. (2014). Sociodemographic and behavioral factors associated with physical activity in Brazilian adolescents. BMC Public Health, 14(1), 485.

deRuiter, W. K., Cairney, J., Leatherdale, S., \& Faulkner, G. (2016). The period prevalence of risk behavior co-occurrence among Canadians. Preventive Medicine, 85, 11-16.

El-ammari, A., \& Bouftini, S. (2017). Level and potential social-ecological factors associated with physical inactivity and sedentary behavior among Moroccan school-age adolescents: a cross-sectional study. Environmental health and preventive medicine, 22(1), 47.

Hallingberg, B. E., Van Goozen, S. H., \& Moore, S. C. (2016). Characteristics associated with risk taking behaviours predict young people's participation in organised activities. Journal of adolescence, 53, 189-194.

Höpker, T., Lampert, T., \& Spallek, J. (2014). Identifizierung und Charakterisierung von Gesundheitsverhaltenstypen bei 11-bis 17-Jährigen Jungen und Mädchen-Eine Clusteranalyse auf Basis der Daten des Kinder-und Jugendgesundheitssurveys (KiGGS). Das Gesundheitswesen, 76(07), 453-461.

Inchley, J., \& Currie, D. (2016). Growing up unequal: gender and socioeconomic differences in young people's health and well-being. Health Behaviour in School-aged Children (HBSC) study: international report from the 2013/2014 survey (No. 7). World Health Organization.

Jáuregui, A., Medina, C., Salvo, D., Barquera, S., \& Rivera-Dommarco, J. A. (2015). Active commuting to school in Mexican adolescents: evidence from the Mexican National Nutrition and Health Survey. Journal of Physical activity and Health, 12(8), 1088-1095.

Lancet Physical Activity Series Working Group. (2012). Global physical activity levels: surveillance progress, pitfalls, and prospects. The lancet, 380(9838), 247-257.

Lipowski, M., Lipowska, M., Jochimek, M., \& Krokosz, D. (2016). Resiliency as a factor protecting youths from risky behaviour: Moderating effects of gender and sport. European journal of sport science, 16(2), 246-255.

McNeill, G., Masson, L. F., Macdiarmid, J. I., Craig, L. C., Wills, W. J., \& Bromley, C. (2017). Socio-economic differences in diet, physical activity and leisuretime screen use among Scottish children in 2006 and 2010: are we closing the gap?. Public health nutrition, 20(6), 951-958.

Palakshappa, D., Virudachalam, S., Oreskovic, N. M., \& Goodman, E. (2015). Adolescent physical education class participation as a predictor for adult physical activity. Childhood Obesity, 11(5), 616-623.

Paudel, S., Subedi, N., Bhandari, R., Bastola, R., Niroula, R., \& Poudyal, A. K. (2014). Estimation of leisure time physical activity and sedentary behaviour among school adolescents in Nepal. BMC public health, 14(1), 637.

Peltzer, K., \& Pengpid, S. (2016). Leisure time physical inactivity and sedentary behaviour and lifestyle correlates among students aged 13-15 in the association of Southeast Asian nations (ASEAN) member states, 2007-2013. International journal of environmental research and public health, $13(2), 217$.

Pereira A.S. et al. (2018). Metodologia da pesquisa científica. [e-book]. Santa Maria. Ed. UAB/NTE/UFSM. Recuperado de: em: https://repositorio.ufsm.br/bitstream/handle/1/15824/Lic_Computacao_Metodologia-Pesquisa-Cientifica.pdf?sequence=1

Santos, C. M., Wanderley Júnior, R. D. S., Barros, S. S. H., Farias Júnior, J. C. D., \& Barros, M. V. G. D. (2010). Prevalência e fatores associados à inatividade física nos deslocamentos para escola em adolescentes. Cadernos de Saúde Pública, 26(7), 1419-1430.

Sergipe, SEED (2016). Secretaria de Estado da Educação de Sergipe. Resumo de Matrículas, 2016. http://www.seed.se.gov.br.

Sousa, T. F. D., José, H. P. M., \& Barbosa, A. R. (2013). Condutas negativas à saúde em estudantes universitários brasileiros. Ciência \& Saúde Coletiva, 18(12), 3563-3575.

Tassitano, R. M., Dumith, S. C., Chica, D. A. G., \& Tenório, M. C. M. (2014). Agregamento dos quatro principais comportamentos de risco às doenças não transmissíveis entre adolescentes. Revista Brasileira de Epidemiologia, 17, 465-478.

Wichstrøm, L., von Soest, T., \& Kvalem, I. L. (2013). Predictors of growth and decline in leisure time physical activity from adolescence to adulthood. Health psychology, 32(7), 775 .

Winkvist, A., Hultén, B., Kim, J. L., Johansson, I., Torén, K., Brisman, J., \& Forslund, H. B. (2015). Dietary intake, leisure time activities and obesity among adolescents in Western Sweden: a cross-sectional study. Nutrition journal, 15(1), 41.

World Health Organization. (2004). Global strategy on diet, physical activity and health.

World Health Organization. (2014). Global status report on noncommunicable diseases 2014 (No. WHO/NMH/NVI/15.1). World Health Organization.

Yang, X., Telama, R., Hirvensalo, M., Tammelin, T., Viikari, J. S., \& Raitakari, O. T. (2014). Active commuting from youth to adulthood and as a predictor of physical activity in early midlife: The Young Finns Study. Preventive medicine, 59, 5-11. 